diagnosed in $4.2 \%$. EEGs showed epileptiform abnormalities in $43 \% ; 8 \%$ were generalized, $16 \%$ focal, and $19 \%$ both. MRIs were abnormal and indicative of the etiology of SE in $8 \%$. Investigation of the cause of SE includes: 1) blood culture and lumbar puncture if there is clinical suspicion of a systemic or CNS infection; 2) AED blood levels: 3) toxicology and metabolic studies when clinically indicated or if etiology is unknown; 4) EEG to check for focal or generalized discharges, or if pseudostatus is suspected, or in diagnosis of nonconvulsive SE; and 5) MRI after SE is treated and stabilized, and if clinically indicated or if etiology is unknown. (Riviello JJ, Ashwal S, Hirtz D, et al. Practice parameter: Diagnostic assessment of the child with status epilepticus (an evidence-based review): Report of the Quality Standards Subcommittee of the American Academy of Neurology and the Practice Committee of the Child Neurology Society. Neurology November (1 of 2) 2006;67:15421550). (Reprints: American Academy of Neurology, 1080 Montreal Ave, St Paul, MN $55116)$.

COMMENT. The authors recommend further prospective studies to determine what factors may precipitate SE in children; the role for routine or selective laboratory investigations; indications and treatment significance of EEG; role of routine or selective $\mathrm{MRI}$; and the frequency, etiology, and prognosis of nonconvulsive SE after control of convulsive SE.

Cognition and electrical SE during sleep (ESES). The duration of ESES and the localization of interictal foci play a major role in the degree and type of cognitive dysfunction following continuous spike-wave activity. ESES interferes with slow-wave activity at the site of the epileptic focus, impairing learning and other cognitive functions. (Tassinari CA, Rubboli G. Epilepsia Nov 2006;47(Suppl 2):40-43).

\title{
SHORT-DURATION ACTH THERAPY FOR WEST SYNDROME
}

Short-term developmental and seizure outcomes were assessed in 7 children, aged $<12$ months, with cryptogenic West syndrome treated with a short-duration (7-12 days) and low-dose synthetic ACTH at Nagoya City Hospital, Japan. All patients had received a trial of one or two AEDs before ACTH. Daily single dose of ACTH was $0.022-0.027 \mathrm{mg} / \mathrm{kg} / \mathrm{day}$ (mean, $0.96 \mathrm{IU} / \mathrm{kg}$ ), and total dose was $0.17-0.28 \mathrm{mg} / \mathrm{kg}$ (mean, $9.0 \mathrm{IU} / \mathrm{kg}$ ). Interval between enset of spasms and initiation of ACTH was 12-105 days (median 14 days). Tonic spasms were controlled in all patients with no serious side effects. Spasms disappeared within 1 week, and hypsarrhythmia resolved within 2 weeks. EEG abnormalities persisted in 2. IQ or developmental quotients of 6 patients were 79-110 at age 2 to 6 years. One patient with a long treatment lag had a developmental quotient of 60 . (Hattori A, Ando N, Hamaguchi K, et al. Short-duration ACTH therapy for cryptogenic West syndrome with better outcome. Pediatr Neurol Dec 2006;35:415-418). (Respond: Dr Hattori, Department of Pediatrics, Nagoya City Hospital, 1 Kawasumi, Mizuho-cho, Mizuho-ku, Nagoya 467-8601, Japan).

COMMENT. Vitamin B6 and AEDs, including clonazepam, valproic acid, and zonisamide, are used before initiating ACTH, because of possible adverse effects of hormone therapy. Delay in intiation of ACTH may result in poor outcome, and a favorable response to very low dose-short duration therapy should favor a recommendation for early trial of 
ACTH. Previous trials of ACTH using relatively low dose regimens have proven effective without serious side effects (Millichap JG, et al. JAMA 1962;182:125; Ito M et al. Pediatr Neurol 1990;6:240-244), whereas high-dose, long-term regimens are associated with frequent serious side effects (Snead OC III. Pediatr Neurol 1990;6:147-150).

\section{LONG-TERM USE OF THE KETOGENIC DIET}

A retrospective chart review of 28 children (15 males, 14 females) treated with the ketogenic diet for epilepsy for more than 6 years was conducted at Johns Hospital Hospital. Response was documented by attendance at clinic and by telephone. Patients were aged 7 to 23 years at follow-up. Diet duration was 6 to 12 years. Seizure frequency was decreased in $90 \%$ patients. Side effects included kidney stones in $7(25 \%)$, skeletal fractures in $6(21 \%)$, and an increase in the number of children with height below the $10^{\text {th }}$ centile from 10 at initiation of the diet to 23 at follow-up $(p=0.001)$. Overall lipid profiles were generally within the normal range, and cholesterol and triglycerides did not increase significantly. (Groesbeck DK, Bluml RM, Kossof EH. Long-term use of the ketogenic diet in the treatment of epilepsy. Dev Med Child Neurol Dec 2006;48:978-981). (Respond: Eric H Kossof MD, Suite 2158, 200 North Wolfe Street, The Johns Hopkins Hospital, Baltimore, MD 21287).

COMMENT. In this report, the efficacy of the ketogenic diet in the treatment of childhood epilepsy was maintained after long-term use, but side effects, especially growth retardation, kidney stones, and fractures, need to be monitored and may be serious.

Several previous reports of long-term use of the diet, or of adults, 40-50 years of age, previously treated with the ketogenic diet have been published, some from the above authors' institution (Livingston S, 1972) and earlier, from the Mayo Clinic where the diet was first introduced (Keith HM, 1963; Wilder RM, 1921). Livingston allayed concerns about the high fat diet and possible cardiac complications in later life; he found no increased evidence of arteriosclerosis, hypertension, ECG abnormalities, or elevated cholesterol levels in the adults examined. He referred to Haddow Keith, my former colleague and mentor regarding use of the diet, and his report of follow-up data on 530 patients with idiopathic epilepsy treated for periods varying up to 36 years. Of 32 followed for 25 to $30+$ years, 24 were well and 8 improved. Of the total of 530 patients, $30 \%$ were completely free of attacks, $24 \%$ were improved, and $39 \%$ were not benefited; $34(6 \%)$ had died, 4 in status epilepticus.

\section{INFECTIOUS DISEASES}

\section{INFLUENZA A AND FEBRILE SEIZURES}

Predisposing factors and characteristics of febrile seizures in children with influenza A infection were studied in children hospitalized with febrile seizures between January and July 2005 at Tuen Mun Hospital, Hong Kong. Of 177 children confirmed with influenza A infection, $34(19.5 \%)$ had febrile seizures. Ages ranged from 0.9 to 6 years (mean 2.69 years); 19 males and 15 females. Age-matched controls had influenza A but no febrile seizures (control $1 ; n=34$ ), and children with febrile seizures who tested negative for influenza (control $2 ; n=34$ ). Mean maximum body temperature of children with febrile 